\title{
Hauttests zur Diagnostik von allergischen Soforttyp-Reaktionen ${ }^{1}$ Leitlinie der Deutschen Gesellschaft für Allergologie und klinischen Immunologie (DGAKI)
}

\author{
Skin Tests for Diagnostics of Allergic Immediate-Type Reactions \\ Guideline of the German Society for Allergology and Clinical Immunology
}

\section{Bibliografie}

DOI http://dx.doi.org/

10.1055/s-0030-1256476

Pneumologie 2011; 65:

484-495 @ Georg Thieme

Verlag KG Stuttgart · New York

ISSN 0934-8387

\section{Korrespondenzadresse}

Priv.-Doz. Dr. Franziska Ruëff

AllergieZentrum,

Klinik und Poliklinik für

Dermatologie und Allergologie Ludwig-Maximilians-Universität

Frauenlobstraße 9-11

80337 München

Franziska.Rueff@med.uni-

muenchen.de in Abstimmung mit

> dem Ärzteverband Deutscher Allergologen (ÄDA)

- dem Berufsverband Deutscher Dermatologen (BVDD)

- der Deutschen Dermatologischen Gesellschaft (DDG)

- der Deutschen Gesellschaft für Hals-Nasen-Ohrenheilkunde und Kopf- und Hals-Chirurgie (DGHHNOKHC)

- der Deutschen Gesellschaft für Pneumologie und Beatmungsmedizin (DGP) und

- der Gesellschaft für Pädiatrische Allergologie und Umweltmedizin (GPA)

\section{Autoren}

F. Ruëff ${ }^{1}$, K.-C. Bergmann², K. Brockow ${ }^{3}$, T. Fuchs ${ }^{4}$, A. Grübl ${ }^{5}$, K. Jung ${ }^{6}$, L. Klimek , H. Müsken ${ }^{8}$, O. Pfaar ${ }^{7}$, B. Przybilla ${ }^{1}$, H. Sitter ${ }^{9}$, W. Wehrmann ${ }^{10}$

\section{Institute}

${ }^{1}$ AllergieZentrum, Klinik und Poliklinik für Dermatologie und Allergologie, Ludwig-Maximilians-Universität, München

2 Allergie-Centrum-Charité/ECARF, Department of Dermatology and Allergy, Charité - Universitätsmedizin Berlin, Berlin

3 Klinik und Poliklinik für Dermatologie und Allergologie, TU München, München

${ }^{4}$ Hautklinik der Georg-August-Universität Göttingen, Bereich Allergologie, Göttingen

5 Kinderklinik München Schwabing - Klinik und Poliklinik für Kinder- und Jugendmedizin, Klinikum Schwabing,

StKM GmbH und Klinikum Rechts der Isar (AöR) der Technischen Universität München, München

${ }^{6}$ Praxis für Dermatologie und Immunologie, Erfurt

7 Zentrum für Rhinologie und Allergologie der Universitäts-HNO-Klinik Mannheim, Wiesbaden

${ }^{8}$ Schwerpunktpraxis für Allergologie und Pneumologie, Institut für pneumologische und allergologische Begutachtungen, Bad Lippspringe

9 Institut für Theoretische Chirurgie, Philipps-Universität Marburg, Marburg

10 Dermatologische Gemeinschaftspraxis, Münster 


\section{Zusammenfassung}

Das Prinzip der Hauttests bei IgE-vermittelter Soforttyp-Allergie besteht darin, das Allergen an die in der Dermis liegenden IgEAntikörper tragenden Mastzellen heranzubringen. Bei Mastzellaktivierung kommt es zu einer Freisetzung von Mediatoren; die im Wesentlichen durch Histamin ausgelöste sichtbare Testreaktion zeigt sich als Quaddel und Rötung.

Die Indikation zum Hauttest ergibt sich bei Verdacht auf eine allergische Erkrankung vom Soforttyp. Systemische Reaktionen bei Hauttests sind sehr selten. Für diesen Fall muss eine Notfallversorgung verfügbar sein. Relative Kontraindikationen sind Hautkrankheiten im Testfeld, ein deutlich beeinträchtigter Allgemeinzustand, schweres, therapeutisch nicht adäquat eingestelltes Asthma bronchiale. Für Tests, die mit einem erhöhten Risiko einer systemischen anaphylaktischen Reaktion behaftet sind, gelten eine Behandlung mit Betablockern oder Schwangerschaft als weitere Kontraindikationen.

Hauttests können in jedem Lebensalter vorgenommen werden, wobei im Säuglings- und Kleinkinderalter die Indikation zurückhaltend $\mathrm{zu}$ stellen ist. Auswaschphasen von das Testergebnis möglicherweise verfälschenden Arzneistoffen sowie eine Refraktärperiode von etwa einer Woche nach einer akuten anaphylaktischen Reaktion sind zu berücksichtigen. Sofern Hauttests in Betracht kommen, ist der Pricktest die Methode der ersten Wahl. Der Intrakutantest ist sensitiver als der Pricktest und soll vor allem dann vorgenommen werden, wenn der Pricktest unauffällig ist.

Die Tests werden an der Volarseite der Unterarme durchgeführt, beim schmerzhafteren Intrakutantest ist auch der weniger empfindliche Rücken geeignet.

Zunächst sollen immer Tests mit standardisierten Extrakten vorgenommen werden. Auf andere Testsubstanzen soll nur dann ausgewichen werden, wenn standardisierte Testallergene nicht verfügbar sind oder Tests mit ihnen nicht weiterführend waren. Kommt es zu einer Testreaktion gegen selbst zubereitetes Testmaterial, so müssen Kontrollpersonen mitgetestet werden, um unspezifische Reaktionen auszuschließen.

Die Ablesung erfolgt nach 15 bis $20 \mathrm{~min}$. Als positive Testreaktion gilt beim Pricktest ein mittlerer Quaddeldurchmesser von $\geq 3 \mathrm{~mm}$, beim Intrakutantest von $\geq 5 \mathrm{~mm}$.

Trotz bestehender allergischer Reaktionslage können Hauttests negativ sein. Im Fall einer positiven Testreaktion sind durch Bezug auf die Anamnese und ggf. durch Provokationstests klinisch relevante von irrelevanten Testreaktionen zu unterscheiden. Aus einer klinisch stummen Sensibilisierung ergibt sich in aller Regel keine praktische Konsequenz.

\section{Abstract \\ $\nabla$}

Skin tests in patients with IgE-mediated immediate type allergy are performed with the intention to establish a contact between allergens and skin mast cells. The latter carry specific IgE antibodies on their surface. If mast cells get activated, mediators (mainly histamine) are released which induce a visible skin reaction (wheal and erythema).

Skin tests are indicated, if an immediate type allergic disease is suspected. Systemic anaphylactic reactions at skin testing are very rare. However, it is necessary to take them into account and to provide emergency treatment. Relative contraindications comprise skin diseases in the test area, poor general condition and insufficiently treated severe asthma. If tests are used, which have a higher risk for a systemic anaphylactic reaction, pregnancy or beta-blocker therapy, are further contraindications.

Skin test application does not depend on patient age. However, in pre-school age tests are reluctantly performed. It is essential to consider the half-life of drugs which may interfere with the test result, and which have to be discontinued early enough before testing. After anaphylactic reactions there may be a refractory period. Therefore, tests should not be done within the first week after such reactions. Skin prick tests are the procedures of first choice, intradermal tests are more sensitive than prick tests. Skin tests are performed at the flexor side of the forearm. As intradermal tests are more inconvenient, testing can be also done at a less susceptible site of the body (upper back).

It is recommended to use standardized test extracts. However, if standardised extracts are not available or do not yield suitable test results, one may switch to other preparations. If the patient shows a positive reaction to a non-standardized substance, control tests should be performed in healthy subjects in order to exclude an unspecific reaction.

The reaction is read after 15 to $20 \mathrm{~min}$. Skin tests are regarded positive if the mean wheal diameter is $\geq 3 \mathrm{~mm}$ at the prick test, and $\geq 5 \mathrm{~mm}$ at the intradermal test.

Skin test results may be negative although patients are allergic. If a skin test is positive, one will have to distinguish reactions, which are clinically relevant, from those, which are not. History and/or challenge tests help to clarify the relevance of a sensitization. Usually, a clinically irrelevant sensitization does not lead to practical consequences. 


\begin{tabular}{lll} 
Inhaltsverzeichnis & \\
1 & Zielsetzung und Entwicklung der Leitlinie & \\
2 & Pathophysiologie der Hauttestreaktion & $\mathrm{xxx}$ \\
3 & Indikationen & $\mathrm{xxx}$ \\
4 & Kontraindikationen & $\mathrm{xxx}$ \\
\hline 5 & Technischer Ablauf & $\mathrm{xxx}$ \\
5.1 & Voraussetzungen & $\mathrm{xxx}$ \\
5.2 & Testzeitpunkt & $\mathrm{xxx}$ \\
5.3 & Einflüsse der Hauttestreaktionen & $\mathrm{xxx}$ \\
5.4 & Testort & $\mathrm{xxx}$ \\
5.5 & Testvorgang & $\mathrm{xxx}$ \\
6 & Methoden & $\mathrm{xxx}$ \\
6.1 & Pricktest & $\mathrm{xxx}$ \\
6.2 & Intrakutantest (Intradermaltest) & $\mathrm{xxx}$ \\
6.3 & Scratch-Test & $\mathrm{xxx}$ \\
6.4 & Reibtest & $\mathrm{xxx}$ \\
6.5 & Epikutantest mit Sofortablesung & $\mathrm{xxx}$ \\
7 & Testmaterial & $\mathrm{xxx}$ \\
7.1 & Kommerziell erhältliche Testsubstanzen & $\mathrm{xxx}$ \\
7.2 & Selbst zubereitete Testsubstanzen & $\mathrm{xxx}$ \\
7.3 & Kontrollen & \\
8 & Ablesung und Dokumentation der Testreaktionen \\
9 & Beurteilung & $\mathrm{xxx}$ \\
\hline & Literatur & $\mathrm{xxx}$ \\
\hline & & $\mathrm{xxx}$
\end{tabular}

\section{Zielsetzung und Entwicklung der Leitlinie}

Die häufigsten IgE-vermittelten allergischen Erkrankungen vom Soforttyp sind die allergische Rhinokonjunktivitis, das allergische Asthma bronchiale und anaphylaktische Reaktionen. Auch dem atopischen Ekzem, manchen gastrointestinalen Erkrankungen und bestimmten Formen der Arzneimittelüberempfindlichkeit können IgE-vermittelte allergische Reaktionen zugrunde liegen. Ursächlich sind oft Allergien auf Umweltstoffe, insbesondere auf Pollen, Milben, Tierepithelien, Nahrungsmittel, Naturlatex, Insektengifte und Arzneistoffe. Die Diagnostik basiert auf Anamnese, klinischer Untersuchung, Hauttests, Nachweis spezifischer IgE-Antikörper im Serum und gegebenenfalls Provokationstests. Bislang steht in Deutschland keine Leitlinie zu Hauttests bei allergischen Soforttyp-Reaktionen zur Verfügung.

Ziel war es, den aktuellen Wissenstand zu einem wesentlichen Instrument in der Diagnostik allergischer Soforttyp-Erkrankungen zusammenzufassen und daraus Empfehlungen für die praktische Anwendung zu erstellen. Diese Leitlinie richtet sich an alle allergologisch tätigen Ärzte, die Hauttests zur Diagnostik allergischer Soforttyp-Erkrankungen (im folgenden Hauttests genannt) durchführen.

Die vorliegende Leitlinie wurde im Auftrag folgender Fachgesellschaften (in alphabetischer Reihenfolge) erstellt: Ärzteverband Deutscher Allergologen (ÄDA), Berufsverbands Deutscher Dermatologen (BVDD), Deutsche Dermatologische Gesellschaft (DDG), Deutsche Gesellschaft für Allergologie und klinische Immunologie (DGAKI), Deutsche Gesellschaft für Hals-NasenOhrenheilkunde und Kopf- und Hals-Chirurgie (DGHHNOKHC), Deutsche Gesellschaft für Pneumologie und Beatmungsmedizin (DGP), Gesellschaft für Pädiatrische Allergologie und Umweltmedizin (GPA).

Die Leitlinie ist entsprechend den methodischen Vorgaben zur Entwicklung von Leitlinien für Diagnostik und Therapie der Arbeitsgemeinschaft der Wissenschaftlichen Medizinischen Fachgesellschaften (AWMF) erstellt worden und entspricht einer S2Leitlinie. Die für die Erstellung der Leitlinie ausgewertete Litera- tur wurde mit Evidenzgraden ( $\bullet$ Tab. 1) versehen. Die publizierten Leitlinien der American Academy of Allergy, Asthma, and Immunology (AAAAI) [7,36] sowie der European Academy of Allergology and Clinical Immunology (EAACI) [10,22] zur Durchführung von Hauttests wurden berücksichtigt.

Das Verfahren zur Konsensbildung lief wie folgt ab. Im Frühjahr 2006 wurden durch die Vorstände des ÄDA, der DDG, der DGAKI, der DGHHNOKHC, der DGP und der GPA Vertreter für eine Expertengruppe benannt. Die Anmeldung als AWMF-Leitlinie erfolgte durch die DGAKI.

Benannt wurden von den jeweiligen Fachgesellschaften: DGAKI

Prof. Dr. Knut Brockow und Priv.-Doz. Dr. Franziska Ruëff

DDG

Prof. Dr. Hans Christian Korting und Prof. Dr. Bernhard Przybilla BVDD

Prof. Dr. Wolfgang Wehrmann

ÄDA

Prof. Dr. Wolfgang Czech, Prof. Dr. Thomas Fuchs und

Priv.-Doz. Dr. Kerstin Jung

GPA

Prof. Dr. Carl Peter Bauer und Dr. Armin Grübl

DGHHNOKHC

Prof. Dr. Ludger Klimek und Priv.-Doz. Dr. Oliver Pfaar

DGP

Prof. Dr. Karl-Christian Bergmann und Dr. Horst Müsken

Es erfolgte eine Literatursuche mittels Medline, wobei nach folgenden Begriffe gesucht wurde: Skin prick test/testing, intradermal test/testing, immediate type allergy, scratch test/testing, rub test/testing, prick-to-prick. Der Entwurf wurde in Konsensuskonferenzen am 13.10.2007 und am 9.4.2008 diskutiert. Diese wurden von Priv.-Doz. Dr. Sitter moderiert und wissenschaftlich begleitet.

Die Leitlinie wird durch Publikationsorgane der allergologischen Fachgesellschaften und in der AWMF-Leitliniensammlung (http://awmf.org) veröffentlicht. Anderen Fachverbänden wird die Leitlinie zur Übernahme empfohlen und interessierten Fachzeitschriften zum Nachdruck zur Verfügung gestellt. Eine Überarbeitung der Leitlinie ist 2014 vorgesehen, verantwortlich ist die Erstautorin.

Tab. 1 Einteilung der Evidenzstärke (nach [43]).

\begin{tabular}{|ll|}
\hline \begin{tabular}{|l|} 
Evidenzgrad \\
Ia
\end{tabular} & $\begin{array}{l}\text { Evidenz durch } \\
\text { Metaanalyse randomisierter, kontrollierter Studien }\end{array}$ \\
\hline 1 b & $\begin{array}{l}\text { randomisierte kontrollierte Studie } \\
\text { gut angelegte, kontrollierte Studie ohne } \\
\text { Randomisierung }\end{array}$ \\
\hline 2 b & $\begin{array}{l}\text { gut angelegte, quasi experimentelle Studie } \\
\text { gut angelegte, nicht experimentelle deskriptive } \\
\text { Studie (z. B. Vergleichsstudie, Korrelationsstudie, } \\
\text { Fall-Kontroll-Studie) }\end{array}$ \\
\hline 4 & $\begin{array}{l}\text { Expertenmeinung, Konsensuskonferenz, } \\
\text { physiologische Modelle, Fallserien }\end{array}$ \\
\hline
\end{tabular}




\section{Pathophysiologie der Hauttestreaktion}

$\nabla$

Reaktionen mit den klinischen Symptomen einer Soforttyp-Allergie können allergische oder nicht allergische (z.B. toxische oder pseudo-allergische) Mechanismen zugrunde liegen. Vorwiegend werden allergische Reaktionen vom Soforttyp durch einen über Immunglobulin E ( $\mathrm{IgE}$ ) vermittelten Mechanismus ausgelöst. IgE-Antikörper sind zytotrop und werden an IgE-Rezeptoren an der Zellmembran gebunden. Kommt es zur Überbrückung von mindestens zwei auf Mastzellen oder basophilen Granulozyten gebundenen spezifischen IgE-Antikörpern durch ein Allergen, wird eine Mediatorfreisetzung ausgelöst. Mediatoren liegen präformiert intrazellulär vor (z.B. Histamin, Tryptase, Tumornekrose-Faktor $\alpha$ ) oder sie werden im Reaktionsablauf neu gebildet (z. B. Prostaglandine, Leukotriene oder Interleukin-4).

Das Prinzip der Hauttests bei IgE-vermittelter Soforttyp-Allergie besteht darin, das Allergen an die in der Dermis vor allem perivaskulär gelagerten Mastzellen heranzubringen. Liegen in der Haut Mastzellen, die spezifische IgE-Antikörper gegen das Testallergen tragen, so kommt es zu deren Aktivierung und Mediatorfreisetzung. Die Mediatoren lösen die sich innerhalb von Minuten entwickelnde Testreaktion aus. Sie entspricht klinisch der durch eine Histamininjektion ausgelösten Lewis-Trias:

- örtliche Rötung durch Vasodilatation

- dermales Ödem durch Steigerung der Kapillarpermeabilität

- Umgebungserythem durch Axonreflex

Klinisch sichtbar sind Rötung (Erythem) und Quaddel (Urtica). Das Maximum der Histaminreaktion tritt innerhalb von 15 min auf [24]. Allergeninduzierte Reaktionen haben ihr Maximum nach 15 bis 20 min. Die Rückbildung erfolgt meist innerhalb von 1 - 2 h. Einige Stunden später können verzögerte Soforttyp-Reaktionen auftreten, die als Quaddel oder als Erythem imponieren. Weiter sind Spättyp-Reaktionen möglich, die sich innerhalb von Stunden bis wenige Tage nach dem Test z.B. als gerötete Papel oder Ekzem zeigen.

\section{Indikationen}

\section{$\nabla$}

Der Verdacht auf eine allergische Erkrankung vom Soforttyp ergibt sich aus der Anamnese und dem klinischen Bild. Hauttests können einen Hinweis auf eine IgE-vermittelte Sensibilisierung geben, der Nachweis spezifischer IgE-Antikörper erfolgt durch In-vitro-Tests [71]

Auch zur Erfassung einer atopischen Diathese durch den Nachweis von Soforttyp-Sensibilisierungen gegenüber verbreiteten Aeroallergenen ist der Test geeignet. Bei der Abklärung vermutlich pseudoallergischer Reaktionen sollte auf Hauttests nicht verzichtet werden, da in Einzelfällen doch ein immunologischer Mechanismus möglich ist.

\section{Kontraindikationen}

Bei der Hauttestung handelt es sich um eine diagnostische Allergenexposition, somit um einen dermalen Provokationstest. Die Kontraindikationen ergeben sich wesentlich durch das Risiko des Auftretens von anaphylaktischen Reaktionen $[8,44,45,70]$. Zwar sind schwere Reaktionen bei Hauttests insgesamt sehr selten $[70,81]$, aber sie können vorkommen und Todesfälle $[6,44]$ infolge von Hauttests wurden beschrieben. Allgemeinsymptome entwickeln sich zumeist innerhalb von 10-20 [45] bzw. 30 min

\section{Tab. 2 Kontraindikationen des Hauttests.}

Hautkrankheit im Testfeld

deutlich beeinträchtigter Allgemeinzustand

instabiles oder therapeutisch nicht adäquat eingestelltes Asthma bronchiale

bei Tests, die mit dem erhöhten Risiko einer systemischen Reaktion behaftet sind: Behandlung mit Betablockern*

Schwangerschaft*

* Ausnahme: Vom Testresultat hängt eine wichtige therapeutische Entscheidung ab und eine systemische anaphylaktische Reaktion ist aufgrund der Gesamtumstände mit hoher Wahrscheinlichkeit nicht zu erwarten.

[44] nach Allergenkontakt, können aber auch später auftreten. Von einer erhöhten Gefährdung auszugehen ist insbesondere bei

- sehr schweren anaphylaktischen Reaktionen in der Anamnese,

- bedeutsamen Beschwerden zum Testzeitpunkt, vor allem Asthma bronchiale [44],

- Allergenkontakt kurz vor dem Test [45],

- Test mit hoch konzentrierten Allergenen [23],

- Intrakutantests [44],

- Test mit nativen Allergenen [20,59],

- Behandlung mit Betablockern [36].

Die im Einzelnen zu beachtenden Kontraindikationen gehen aus - Tab. 2 hervor. Bestehen Kontraindikationen oder erscheint aus anderen Gründen ein Hauttest nicht zumutbar, sollten zunächst In-vitro-Tests vorgenommen werden, sofern für das betreffende Allergen verfügbar. Es ist im Einzelfall zu prüfen, ob danach noch Hauttests erforderlich sind. Ist ein Hauttest trotz Kontraindikationen angezeigt, so ist dies zu begründen und in der Patientenakte zu dokumentieren.

\section{Technischer Ablauf}

$\nabla$

\subsection{Voraussetzungen}

Vor einem Hauttest sind eine eingehende allergologische Anamnese zu erheben und eine dem jeweiligen Krankheitsbild angepasste klinische Untersuchung durchzuführen. Die sich daraus ergebende Verdachtsdiagnose ist zu dokumentieren. Es ist zu entscheiden, welche Hauttests erforderlich sind. Weiter ist $\mathrm{zu}$ prüfen, ob Kontraindikationen vorliegen. Die Eignung des Patienten zum Hauttest ist vor jeder Testung festzustellen.

Der Patient oder ggf. seine Sorgeberechtigen ist bzw. sind über Art und Ablauf der Tests, über die zu erwartenden Reaktionen und die in Einzelfällen möglichen systemischen Symptome zu informieren. Die Aufklärung sollte dokumentiert werden. Bei erhöhtem Risiko von Allgemeinreaktionen wird eine schriftliche Einverständniserklärung des Patienten bzw. der Sorgeberechtigten empfohlen.

Wenn anamnestisch sehr schwere anaphylaktische Symptome aufgetreten sind und In-vitro-Verfahren nicht weiterführend waren, so wird der Test mit den verdächtigen Allergenen vorzugsweise unter stationärer Überwachung vorgenommen; in Einzelfällen kann Notfallbereitschaft angezeigt sein. Durch einen Schwellentest - Verdünnung der Testlösung und schrittweise Tests mit ansteigenden Allergenkonzentrationen - kann das Risiko einer Allgemeinreaktion weiter gesenkt werden.

Ein Asthma bronchiale ist vor einem Test optimal einzustellen (FEV1 [forcierte Einsekundenkapazität] >70\% Sollwert). Bei schlechter Lungenfunktion sollten vor und über sechs Stunden nach der Testung Peakflow-Kontrollen vorgenommen werden. 
Tab. 3 Notfallausrüstung zur Behandlung systemischer Reaktionen (modifiziert nach [73]).

Stethoskop, Blutdruckmessgerät
Stauschlauch, Spritzen, Venenverweilkanülen, Infusionsbesteck
Sauerstoff mit Maske/Brille
Guedel-Tubus, Beatmungsbeutel, Absaugvorrichtung, Intubations-
besteck und -tuben
Injektionsnadeln
Adrenalin zur Injektion
$\mathrm{H}_{1}$ - und $\mathrm{H}_{2}$-Antihistaminika zur intravenösen Injektion
Infusionslösungen (physiologische $\mathrm{NaCl}-$ Elektrolytlösungen, kolloidale
Lösungen)
Glukokortikosteroide zur intravenösen Injektion
Bronchodilatator (rasch wirksames $\beta_{2}$-Mimetikum zur Inhalation)
automatischer externer Defibrillator (nicht in der Pädiatrie)

Arzt und Assistenzpersonal müssen im Fall testinduzierter Allgemeinreaktionen in der Lage sein, solche Notfälle adäquat zu behandeln [73]. Die erforderliche medikamentöse und apparative Mindestausrüstung zur Behandlung von anaphylaktischen Reaktionen ist in $\odot$ Tab. 3 aufgelistet.

\subsection{Testzeitpunkt}

Wenn Kontraindikationen ( $\bullet$ Tab. 2) vorliegen, die das Risiko einer anaphylaktischen Reaktion erhöhen können, ist abzuwarten bzw. der Patient zu behandeln, bis der Test vorgenommen werden kann. Die Auswaschphase von Arzneistoffen, die die Hautreaktivität hemmen ( $\odot$ Tab. 4), ist zu beachten.

Bei der Diagnostik anaphylaktischer Reaktionen, z. B. durch Insektenstiche, Nahrungsmittel oder Arzneimittel, ist zu beachten, dass Tests nicht innerhalb einer möglichen Refraktärperiode nach dem letzten akuten Ereignis stattfinden sollten [28]. Es wird empfohlen, frühestens eine Woche nach einem akuten Ereignis zu testen und den Test dann nochmals etwa drei bis vier Wochen nach dem Ereignis zu wiederholen, falls das Ergebnis des ersten Tests zweifelhaft ist. Bei inhalativen allergischen Beschwerden kann grundsätzlich auch bei leichten bis mittelschweren aktuellen Beschwerden getestet werden.

Hauttests können unabhängig vom Lebensalter des Patienten, also auch bei Säuglingen und Kleinkindern, durchgeführt werden. Zwar gibt es Hinweise, dass die Hauttestreaktivität bei Säuglingen und Kleinkindern [78] sowie älteren Patienten geringer als bei Erwachsenen in mittlerem Lebensalter ist $[39,78]$, verwertbar sind die Hauttests aber dennoch.

\begin{tabular}{|c|c|c|}
\hline Arzneistoff & Unterdrückung* & Absetzen vor Test \\
\hline \multicolumn{3}{|l|}{ Antihistaminika } \\
\hline H1-Blocker & +++ & $>3$ Tage \\
\hline \multicolumn{3}{|l|}{ Länger wirksame H1-Blocker } \\
\hline Astemizol & +++ & $>8$ Wochen \\
\hline H2-Blocker & $\varnothing 1+$ & $\varnothing$ \\
\hline \multicolumn{3}{|l|}{ Mastzellstabilisatoren } \\
\hline Cromoglicinsäure, Nedocromil & $\varnothing$ & $\varnothing$ \\
\hline Ketotifen & +++ & $>5$ Tage \\
\hline \multicolumn{3}{|l|}{ Glukokortikoide $^{2}$} \\
\hline Topisch > 4 Wochen (im Testareal) [67] & + & $>1$ Woche $^{1}$ \\
\hline Inhalativ & $\varnothing$ & $\varnothing$ \\
\hline \multicolumn{3}{|l|}{ Systemisch/Kurzzeit } \\
\hline$<50$ mg/d Prednisolon-Äquivalent & $\varnothing$ & $>3$ Tage $^{3}$ \\
\hline > 50 mg/d Prednisolon-Äquivalent & $\varnothing /(+)$ & $>1$ Woche $^{3}$ \\
\hline Systemisch/Langzeit & $(+)$ & \\
\hline$<10 \mathrm{mg} / \mathrm{d}$ Prednisolon-Äquivalent & $\varnothing$ & $\varnothing$ \\
\hline$>10$ mg/d Prednisolon-Äquivalent & $\varnothing$ & $>3$ Wochen $^{3}$ \\
\hline \multicolumn{3}{|l|}{ Broncholytika } \\
\hline Terbutalin [64] & $\varnothing$ & $\varnothing$ \\
\hline Bambuterol [64] & $\varnothing$ & $\varnothing$ \\
\hline Salmeterol [63] & $\varnothing$ & $\varnothing$ \\
\hline Salbutamol [63] & $\varnothing$ & $\varnothing$ \\
\hline Theophyllin & $\varnothing$ & $\varnothing$ \\
\hline \multicolumn{3}{|l|}{ Psychopharmaka } \\
\hline \multicolumn{3}{|l|}{ (z. B. Imipramin, Amitriptylin, Desipramin) } \\
\hline Neuroleptikum (Promethazin) & ++ & $>5$ Tage \\
\hline $\begin{array}{l}\text { Selektive Serotonin-Wiederaufnahmehemmer } \\
\text { (z.B. Fluoxetin, Sertralin) }\end{array}$ & $\varnothing$ & $\varnothing$ \\
\hline$\beta$-adrenerge Agonisten $[1,79]$ & $\varnothing$ & $\varnothing$ \\
\hline \multicolumn{3}{|l|}{ Sonstige systemisch angewandte Arzneistoffe } \\
\hline Leukotrien-Rezeptorantagonisten $[16,32]$ & $\varnothing$ & $\varnothing$ \\
\hline Cyclosporin A [54] & $\varnothing$ & $\varnothing$ \\
\hline Intravenöse Immunglobuline [50] & + & $?$ \\
\hline \multicolumn{3}{|c|}{$\begin{array}{l}\text { * Unterdrückung des Hauttests: } \varnothing \text {, keine Hinweise; }(+) \text {, möglich, +, gering; ++, mittelgradig, +++, stark; ?, fraglich. } \\
{ }^{1} \text { Abhängig von der Wirkstärke des angewandten Präparates und Anwendungsdauer bis zu >3 Wochen. } \\
{ }^{2} \text { Dosisangaben für Erwachsene. } \\
{ }^{3} \text { In einer retrospektiven Studie keine Beeinflussung der Hauttestreagibilität durch } 10 \text { bis } 60 \text { mg Prednison für } 2 \text { oder mehr } \\
\text { Jahre [19]. }\end{array}$} \\
\hline
\end{tabular}

Tab. 4 Beeinflussung von Soforttyp-Reaktionen durch Arzneistoffe (nach $[18,40,47,82]$ ). 


\subsection{Einflüsse auf Hauttestreaktionen}

Die Wirkung systemisch oder topisch am Testort angewandter Arzneimittel, die Hauttestreaktionen vom Soforttyp inhibieren können, ist zu beachten ( $\checkmark$ Tab.4). Zu der möglichen Beeinflussung von Hauttests durch Arzneistoffe ist festzustellen, dass vielfach formale Studien fehlen, somit keine eindeutige Evidenzlage besteht und daher die in Tab. 4 gegebenen Empfehlungen nur Anhaltspunkte sind.

Grundsätzlich sollen Arzneimittel, die Testreaktionen unterdrücken können, abgesetzt werden; nach Abklingen ihrer Wirkung wird zu einem späteren Zeitpunkt getestet. Im Einzelfall kann es allerdings erforderlich sein, Tests trotz Anwendung solcher Arzneimittel durchzuführen. Falls hierbei eindeutige Soforttyp-Reaktionen auf die Histaminkontrolle auftreten, so sind auch die Reaktionen auf die Allergenextrakte verwertbar [14]. Falls es sich um Arzneistoffe handelt, die eine mastzellstabilisierende Wirkung haben, ist eine positive Histaminkontrolle kein Beleg für die Verlässlichkeit des Tests. Ist die Histaminkontrolle negativ, so sind die Tests zu einem späteren Zeitpunkt zu wiederholen.

Zwar inhibieren topische Glukokortikosteroide die Hauttestreaktivität erst nach mehrwöchiger Anwendung [66], jedoch erfolgt eine solche Behandlung nur bei Krankheitserscheinungen im Testfeld, die ihrerseits bereits ein Testhindernis darstellen.

Die Anwendung eines Oberflächenanästhetikums (z.B. EMLA ${ }^{\circledR}$; Wirkstoffe: Lidocain und Prilocain) kann zu einer Abschwächung der Testreaktion führen, wobei dies vor allem für das Erythem, weniger für die Quaddelbildung zutrifft $[66,77]$. Andere Autoren sahen keine bedeutsame Änderung der Hauttestergebnisse durch eine Vorbehandlung mit EMLA ${ }^{\circledR}[3]$.

Es ist davon auszugehen, dass die Hauttestreaktivität auch durch Faktoren wie z.B. die psychische Befindlichkeit beeinflusst werden kann [84]. Systematische Untersuchungen zum Effekt anderer möglicher modifizierender Faktoren, z.B. Ernährung, Nahrungsergänzungsmittel, pflanzliche Medikamente, Hormone oder Infekte, fehlen.

\subsection{Testort}

Die Pricktests werden an der Volarseite der Unterarme durchgeführt, ersatzweise kann an der Haut des oberen Rückens getestet werden. Für den Intrakutantest ist der Rücken zu bevorzugen, da das Schmerzempfinden dort geringer ausgeprägt ist. Dabei ist zu beachten, dass der Durchmesser der Hauttestreaktionen am Rücken größer ausfällt als am Unterarm $[56,75]$. Weiter sind die Reaktionen am Rücken im oberen Drittel kleiner als im unteren Drittel [55].

Der Testort wird mit einem Stift so markiert, dass die einzelnen Reaktionen bei Ablesung der jeweiligen Testzubereitung eindeutig zuzuordnen sind. Der Abstand zwischen den einzelnen Testorten sollte ausreichend sein [7,42]. Bei zu geringem Abstand können ausgeprägte Testreaktionen zu falsch positiven Ergebnissen in der Umgebung führen bzw. nah benachbarte, ineinanderlaufende Reaktionen nicht mehr eindeutig ablesbar sein.

Wenn die Haut mit Externa behandelt worden ist, kann es zu einem Verlaufen der Testlösungen kommen, sodass diese vor dem Test nicht verwendet werden sollen bzw. ggf. abgewischt werden müssen.

\subsection{Testvorgang}

Der Test selbst erfolgt zügig, um weitgehend gleiche Ablesezeitpunkte für die Einzeltests zu gewährleisten. Von Testbeginn bis zur Ablesung der Testreaktionen nach 15-20 min bleibt der Patient im Aufsichtsbereich des Arztes, mit der Maßgabe, bei Auf- treten über den Testort hinausreichender Symptome unverzüglich Bescheid zu geben. Ist die Erfassung von verzögerten Reaktionen oder Spättyp-Reaktionen diagnostisch erforderlich, so wird der Patient zu diesen Ablesungen wieder einbestellt; auf eine zuverlässige Markierung der Testorte ist dann zu achten. Im übrigen wird der Patient darüber informiert, dass später aufgetretene Reaktionen bei Wiedervorstellung berichtet werden sollen. Sind aufgrund der Anzahl der zu testenden Allergene mehrere Sitzungen erforderlich und traten bei der vorhergehenden Testung Reaktionen im Testfeld auf, so sollte das Intervall bis zum nächsten Test etwa 2 Tage betragen. Falls der vorangehende Hauttest unauffällig war, kann bereits nach 1 Tag weiter getestet werden.

\section{Methoden \\ $\nabla$}

\subsection{Pricktest}

Ein Tropfen der Testlösung wird mit einer Pipette oder einem anderen Applikator auf die Haut aufgebracht. Durch den Tropfen hindurch wird die Haut oberflächlich angestochen, eine Blutung sollte dabei nicht auftreten.

Es wird empfohlen, den Pricktest mit einer speziellen Lanzette mit $1 \mathrm{~mm}$ Spitzenlänge auszuführen („Puncture-Test“). Die Nadel wird mit leichtem Druck durch die Testlösung hindurch kurz senkrecht in die Haut gedrückt. Vorteile dieses Vorgehens sind eine geringere Traumatisierung und bessere Reproduzierbarkeit im Vergleich zum Pricktest, der mit einer Blutlanzette durchgeführt wird [61].

Eine Variante des Pricktests wird mit Blutlanzetten aus Metall mit langer Spitze vorgenommen. Mit der Lanzette wird die Haut durch den Tropfen hindurch in einem Winkel von etwa $45^{\circ}$ angestochen und kurz angehoben. Verschiedene Lanzettentypen aus Metall erbrachten annähernd gleiche Ergebnisse [2,69]; in einer anderen Untersuchung zeigte sich in Abhängigkeit von der Länge der Nadelspitze ein größerer Durchmesser der Histaminquaddel bei größerer Traumatisierung der Haut [55]. Plastiklanzetten [9] und Lanzetten mit einem bürstenartigen Kopf [12] erbringen schlechter reproduzierbare Ergebnisse.

In den Händen geübter Untersucher sind der Pricktest mit einer Blutlanzette und der Pricktest mit einer speziellen Pricktestnadel („Puncture-Test“) als gleichwertig anzusehen [61]. Insgesamt ist davon auszugehen, dass die Art der verwendeten Nadel die Technik des Hauttests und die Testergebnisse beeinflussen [7].

Grundsätzlich wird empfohlen, für die Testung jedes einzelnen Allergens einer Pricktestreihe bei einem Patienten eine neue Lanzette zu verwenden [65]. Zwar wird vielfach in der klinischen Routine für eine ganze Prickserie an einem Patienten nur eine Lanzette verwendet. Es wird dazu auch die Auffassung vertreten, dass ein Abwischen der Lanzette mit einem Tupfer unter festem Druck nach jedem einzelnen Test ausreichend sei. Es besteht aber bei Testung mit nur einer Lanzette das Risiko der Allergenverschleppung. Dieser Verdacht muss dann geäußert werden, wenn nach einer ausgeprägten Reaktion eine oder mehrere schwächere Reaktionen auftreten. Hier wird dann ggf. eine Wiederholung einzelner Tests erforderlich.

Pricktests können auch als „Multitests“ durchgeführt werden. Dabei wird ein kommerziell erhältlicher Stempel mit allergenbeschichteten Testnadeln kurz in die Haut gedrückt. Sensitivität und Reproduzierbarkeit derartiger Tests erwiesen sich in einer Studie als schlecht [2], in anderen war die Reproduzierbarkeit 
gut $[5,25]$. Die Sensitivität von Pricktests mit allergenbeschichteten Nadeln war gut $[13,38]$.

Aufgrund der guten Reproduzierbarkeit der Testergebnisse, des geringen Risikos systemischer Reaktionen und einer günstigen Korrelation mit der klinischen Reagibilität ist der Pricktest als Hauttestmethode der ersten Wahl anzusehen $[7,17,47]$.

\subsection{Intrakutantest (Intradermaltest)}

Die Testlösung wird in eine sterile Tuberkulinspritze aufgezogen, mit einer 21er-Kanüle werden 0,02-0,05 ml davon streng intrakutan injiziert. Mit dem injizierten Volumen soll an der Hautoberfläche eine im Durchmesser etwa $3 \mathrm{~mm}$ große Quaddel gesetzt werden. Die Testlösung muss steril und zur intrakutanen Anwendung geeignet sein.

Für jeden einzelnen Test an einem Patienten müssen eine neue Spritze und eine neue Kanüle verwendet werden. Ein Wechsel der Kanüle alleine ist nicht ausreichend, da es bei der Injektion zu einem Zurücklaufen der Flüssigkeit und damit zu einer Kontamination der Spritze kommen kann $[33,41,46]$.

Einige Untersuchungen haben für Prick- und Intrakutantests eine gleich gute Sensitivität gezeigt, sofern für den Pricktest ausreichend hohe Testkonzentrationen verwendet wurden $[35,26]$. Allerdings konnten in anderen Studien im Pricktest nicht erfassbare Sensibilisierungen mit dem Intrakutantest nachgewiesen werden [52,83] bzw. der Durchmesser der Hauttestreaktionen war im Intrakutantest größer [34].

Somit ist der Vorteil des Intrakutantests gegenüber dem Pricktest eine höhere Sensitivität, Nachteile sind Schmerzhaftigkeit, höherer technischer und zeitlicher Aufwand und ein höheres Risiko systemischer Reaktionen [44].

\subsection{Scratch-Test}

Zum Einbringen des Allergens in die Haut des Unterarms wird eine etwa $5 \mathrm{~mm}$ lange oberflächliche, nicht blutende Skarifikation gesetzt und die Testlösung danach aufgetropft. Beim „Scratch-Chamber-Test“ wird das Testareal anschließend für 20 min von einer Testkammer bedeckt. Die Ergebnisse sind sehr von der individuellen Geschicklichkeit des Untersuchers abhängig und damit schlecht standardisierbar und reproduzierbar. Von Nachteil sind häufig unspezifische oder schwer interpretierbare Reaktionen aufgrund der im Vergleich zum Pricktest größeren mechanischen Irritation [15], auch ist das Risiko systemischer Reaktionen erhöht [80]. Belege für eine höhere Sensitivität des „Scratch-Chamber-Tests“ bei der Testung von Aeroallergenen fanden sich nicht [60].

\subsection{Reibtest}

Beim Reibtest wird zumeist natives Material unter Ausübung leichten Druckes mehrmals (zumeist 10-mal) auf einem etwa $5 \times 5 \mathrm{~cm}$ großen Areal in die Haut gerieben [29]. Der Test ist auch zur Diagnose einer Kontakturtikaria geeignet. Bei Fehlen einer Reaktion am üblichen Testort (zumeist Unterarminnenseite) kann er manchmal in früher erkrankten Arealen (beispielsweise Handrücken) positiv ausfallen. Vorteile des Reibtests sind die geringe Belastung des Patienten und die hohe Spezifität, Nachteil eine geringe Sensitivität.

\subsection{Epikutantest mit Sofortablesung}

Üblicherweise wird beim Epikutantest das Testmaterial 24 bis $48 \mathrm{~h}$ belassen [76]. Beim Epikutantest mit Sofortablesung wird das Testmaterial offen oder unter einer gebräuchlichen Epikutantestkammer okklusiv für 20 min auf die Haut aufgebracht und danach entfernt. Die Methode erlaubt Tests mit unterschiedlichem, auch wenig oder nicht aufbereitetem Material, z. B. Flüssigkeiten, Salbenzubereitungen oder festen Gegenständen. Epikutantests mit Sofortablesung sollten vor allem dann vor einem Pricktest durchgeführt werden, wenn schwere Reaktionen auf ein Allergen aufgetreten sind, das in Ermangelung standardisierter Testextrakte nativ getestet werden muss [23].

\section{Testmaterial}

$\nabla$

\subsection{Kommerziell erhältliche Testsubstanzen}

Kommerzielle Testextrakte von zahlreichen Allergenen werden von verschiedenen Herstellern angeboten. Zwischen den Produkten einzelner Hersteller können deutliche Unterschiede hinsichtlich der Auslösung von Testreaktionen bestehen. Die Standardisierung von Allergenlösungen wird meist in Bezug auf eine $1 \%$ ige oder eine $0,1 \%$ ige Histaminlösung vorgenommen. Eine 10-fach höhere Konzentration von Histamin oder Allergen erhöht den Quaddeldurchmesser etwa um den Faktor 1,5 [21], entsprechend werden Testreaktionen in Bezug auf eine mit 0,1\%iger Histaminlösung standardisierte Allergenkonzentrationen im Durchschnitt schwächer ausfallen, als wenn der Bezug auf eine $1 \%$ ige Histaminlösung erfolgt.

Die Zusammensetzung und Standardisierung der Testlösungen sind entscheidend für die Sensitivität, Spezifität und Reproduzierbarkeit der Testergebnisse. Es sind vorzugsweise qualitativ und quantitativ immunchemisch sowie biologisch standardisierte Extrakte zu verwenden [7,48]. Zumeist handelt es sich um wässrige Lösungen, daneben sind vor Gebrauch zu rekonstituierende Lyophilisate erhältlich. Die Allergenkonzentration von Lösungen für den Intrakutantest liegt üblicherweise um den Faktor 100 bis 1000 niedriger als bei Pricktestlösungen [7], je nach Allergen kann der Verdünnungsfaktor zwischen 1:10 bis zu $1: 30000[21,34,35]$ betragen.

Die Testsubstanzen müssen im Kühlschrank bei einer Temperatur von $2-8{ }^{\circ} \mathrm{C}$ und einer optimalen mittleren Temperatur von $4^{\circ} \mathrm{C}$ aufbewahrt werden. Sowohl eine zu warme Lagerung wie ein Einfrieren der Testsubstanzen führen zu einem Verlust der allergenen Potenz [57]. Aber auch bei korrekter Lagerung kommt es im Laufe der Zeit zu einer Minderung der biologischen Aktivität [82]. Die Verfallsdaten der Testlösungen sind zu beachten.

Insbesondere die Ergebnise von Tests mit selteneren Allergenen sowie instabilen Allergenen (z. B. viele Nahrungsmittelallergene) sind kritisch zu werten. Auf Tests mit kommerziellen Extrakten sollte jedoch nicht grundsätzlich verzichtet werden, da diese einfacher zu handhaben sind.

Es hat sich bewährt, für bestimmte Fragestellungen feste Testreihen zusammenzustellen. Die Auswahl von standardmäßig zu testenden Aeroallergenen muss regionale Gegebenheiten berücksichtigen. Ein Vorschlag für eine Standardreihe zur Basisdiagnostik bei Patienten mit Soforttyp-Reaktionen oder anderen dem atopischen Formenkreis zuzuordnenden Erkrankungen findet sich in Tab. 5, eine Nahrungsmittelstandardreihe in Tab. 6. Angesichts der engen Beziehungen zwischen allergischen Reaktionen auf Nahrungsmittel und auf Aeroallergene sind bei Verdacht auf eine Nahrungsmittelüberempfindlichkeit Aeroallergene stets mitzutesten. Tests mit Standardreihen werden unter Berücksichtigung der Anamnese fallweise durch Testung von zusätzlichen Testblöcken oder von Einzelallergenen ergänzt. 
Tab. 5 Klinisch wichtige Aeroallergene* für den Hauttest bei Erwachsenen.

\begin{tabular}{|c|c|}
\hline Pollen & $\begin{array}{l}\text { Beifußa }^{\mathrm{a}} \\
\text { Birke }^{\mathrm{a}} \\
\text { Erle }^{\mathrm{a}} \\
\text { Esche } \\
\text { Gräser }^{\mathrm{a}} \\
\text { Hasel }^{\mathrm{a}} \\
\text { Olive }^{\mathrm{a}} \\
\text { Parietaria (Glaskraut) a }^{\mathrm{a}} \\
\text { Platane }^{\mathrm{a}} \\
\text { Ragweed (Beifuß-Ambrosie) a }^{\text {a }} \\
\text { Wegerich }_{\text {Zypresse }}\end{array}$ \\
\hline Schimmelpilze & $\begin{array}{l}\text { Alternaria alternata }{ }^{a} \\
\text { Aspergillus fumigatus } \\
\text { Cladosporium herbarum }\end{array}$ \\
\hline $\begin{array}{l}\text { Milben, Epithelien } \\
\text { und anderes }\end{array}$ & $\begin{array}{l}\text { Dermatophagoides farinae } \\
\text { Dermatophagoides pteronyssinus }^{\mathrm{a}} \\
\text { Hundehaare/-schuppen }^{\mathrm{a}} \\
\text { Katzenhaare/-schuppen }^{\mathrm{a}} \\
\text { Naturlatex } \\
\text { Acarus siro } \\
\text { Lepidoglyphus destructor } \\
\text { Tyrophagus putrescentiae }\end{array}$ \\
\hline Kontrollen & $\begin{array}{l}\mathrm{NaCl} 0,9 \% \\
\text { Histamindihydrochlorid 1,0\% }\end{array}$ \\
\hline \multicolumn{2}{|c|}{$\begin{array}{l}\text { *Die Auswahl von standardmäßig zu testenden Allergenen des Außenbe- } \\
\text { reichs hängt von regionalen Gegebenheiten ab. Weiter können je nach } \\
\text { Anamnese vorwiegend im Innen- bzw. Außenbereich vorkommende Aller- } \\
\text { gene teilweise oder ganz ausgelassen werden. } \\
\text { Weitere Testreihen bedeutsamer Aeroallergene (nach Anamnese zu tes- } \\
\text { ten), z. B. weitere Tierallergene, Vorratsmilben, Schimmelpilze, Kräuter- } \\
\text { und Baumpollen, Gartenblumen. } \\
\text { a Europäische Standardserie [30]. }\end{array}$} \\
\hline
\end{tabular}

\begin{tabular}{l} 
Apfel \\
Banane \\
Erdnuss \\
\hline Forelle \\
Haselnuss \\
Hühnerei \\
Hühnerfleisch \\
Kabeljau \\
Kartoffel \\
Kuhmilch \\
\hline Reiskorn \\
Rindfleisch \\
Roggenmehl \\
Schweinefleisch \\
Sellerie \\
Sojabohne \\
Tomate \\
\hline Weizenmehl \\
Kontrollen \\
NaCl 0,9\% \\
Histamindihydrochlorid 1,0\% \\
Weitere Testreihen von bedeutsamen Nahrungsmit- \\
telallergenen (nach Anamnese zu testen): Backwaren, \\
Ei, Gemüse, Genussmittel, Gewürze und Kräuter, \\
Fische und Meeresfrüchte, Früchte, Milch, Nüsse und \\
Saaten.
\end{tabular}
rungsmittelstandardreihe (Erwachsene).
Tab. 7 Klinisch wichtige Allergene für den Hauttest* bei Kindern.

\begin{tabular}{|c|c|}
\hline Pollen & $\begin{array}{l}\text { Lieschgras } \\
\text { Birke } \\
\text { Beifuß } \\
\text { Ragweed (Beifußambrosie) }\end{array}$ \\
\hline Schimmelpilze & $\begin{array}{l}\text { Aspergillus fumigatus } \\
\text { Alternaria alternata } \\
\text { Cladosporium herbarum } \\
\text { Penicillium notatum }\end{array}$ \\
\hline $\begin{array}{l}\text { Milben, Epithelien, } \\
\text { Verschiedenes }\end{array}$ & $\begin{array}{l}\text { Dermatophagoides pteronyssinus } \\
\text { Dermatophagoides farinae } \\
\text { Hundehaare/-schuppen } \\
\text { Katzenhaare/-schuppen } \\
\text { Pferdehaare/-schuppen }\end{array}$ \\
\hline Nahrungsmittel & $\begin{array}{l}\text { Erdnuss } \\
\text { Hühnerei } \\
\text { Kuhmilch } \\
\text { Sojabohne } \\
\text { Weizenmehl } \\
\text { Haselnuss } \\
\text { Kabeljau }\end{array}$ \\
\hline Kontrollen & $\begin{array}{l}\mathrm{NaCl} 0,9 \% \\
\text { Histamindihydrochlorid 1,0\% }\end{array}$ \\
\hline $\begin{array}{l}\text { *Die in der Tabelle farb } \\
\text { schlag für eine Standa } \\
\text { Anamnese Allergene } \\
\text { deren aufgeführten b. } \\
\text { testet werden. }\end{array}$ & $\begin{array}{l}\text { hobenen Allergene stellen einen Vor- } \\
\text { Kindern dar, bei der je nach individueller } \\
\text { werden können bzw. umgekehrt die an- } \\
\text { och weitere verdächtige Allergene ge- }\end{array}$ \\
\hline
\end{tabular}

Eine Standardreihe für Kinder ist in $\bullet$ Tab. 7 dargestellt. Je nach Anamnese können Allergene weggelassen werden oder zusätzlich getestet werden. Bei jüngeren Kindern wird im allgemeinen nur mit einigen wenigen, gezielt ausgewählten Allergenen getestet; Intrakutantests sind bei Kindern zu vermeiden. Jugendliche können dagegen schon mit der Standardreihe für Erwachsene getestet werden.

\subsection{Selbst zubereitete Testsubstanzen}

Zunächst sollten immer Tests mit standardisierten oder, falls nicht verfügbar, kommerziell erhältlichen nicht standardisierten Extrakten vorgenommen werden. Tests mit selbst aufbereitetem oder nativem Material werden nur dann vorgenommen, wenn kommerzielle Allergenzubereitungen für den Hauttest und Invitro-Tests nicht verfügbar sind bzw. die Tests mit ihnen nicht zu einem diagnostisch verwertbaren Ergebnis geführt haben. Weiter soll auf nicht selbst zubereitete Testsubstanzen nur dann ausgewichen werden, wenn vom Ergebnis des Hauttests eine wichtige diagnostische Zuordnung oder eine therapeutische Entscheidung abhängt.

Von zahlreichen Allergenen sind keine Testzubereitungen auf dem Markt. Besonders gegen Nahrungsmittelallergene ist mit den verfügbaren kommerziellen Zubereitungen eine Sensibilisierung nicht immer nachweisbar. Dann empfiehlt sich eine Testung mit nativem Material, bei der dann oft doch eine Reaktion im Hauttest erzeugt werden kann $[68,74]$. Bei solchen Tests ist folgendes zu berücksichtigen:

- Die geeigneten Testkonzentrationen sind im allgemeinen unbekannt. Zur Vermeidung von überschießenden örtlichen sowie von Allgemeinreaktionen sind, insbesondere bei schweren Reaktionen in der Anamnese oder bei Tests mit potenten Allergenen, Schwellentests mit ansteigenden Allergenkonzentrationen angezeigt. 
- Bei Auftreten einer Reaktion sind zur Überprüfung der Spezifität Kontrolltests an weiteren Personen erforderlich, die darüber aufgeklärt werden müssen und deren Einverständnis einzuholen ist. Sind solche Kontrolltests nicht möglich, so ist der diagnostische Wert der Hauttestreaktion unklar; auf solche Tests ist daher gegebenenfalls von vornherein zu verzichten.

- Für Intrakutantests können nur sterilisierte und pyrogenfreie Zubereitungen verwendet werden. Von manchen Arzneimitteln existieren sterile Lösungen zur intravenösen Gabe, die zur Hauttestung eingesetzt werden können.

- Insbesondere bei Intrakutantests mit nicht standardisiertem Material ist das Risiko der testinduzierten Sensibilisierung zu berücksichtigen. Zumindest im Tierexperiment wird die intrakutane Zufuhr von Allergenen genutzt, um bei Versuchstieren eine Sensibilisierung zu erzeugen $[27,49]$. Kontrolltests an anderen Personen sind mit solchen Substanzen nicht möglich.

- Mit Suspensionen oder stark gefärbten Lösungen können keine Intrakutantests vorgenommen werden. Das Risiko persistierender Pigmentierungen durch farbstoffhaltiges Testmaterial ist auch bei anderen Testformen zu berücksichtigen.

- Mit Stoffen, von denen in der angewandten Menge bzw. Konzentration eine systemische oder örtliche bedeutsame toxische Wirkung zu erwarten ist, kann nicht getestet werden.

- Gemäß 15. Novelle des Arzneimittelgesetzes §2 Abs. 1 handelt es sich bei Testsubstanzen für den Hauttest um Arzneimittel. Die sich daraus ergebenden Folgerungen sind in den einzelnen Bundesländern unterschiedlich geregelt.

Flüssige Allergenträger, beispielsweise Getränke, Injektionslösungen oder Sperma, sind für Hauttests nach gegebenenfalls erforderlicher Verdünnung unmittelbar geeignet. Aus flüssigkeitshaltigen Gegenständen wie z. B. Früchten oder Fleisch kann durch Auspressen oder Pürieren Flüssigkeit gewonnen werden. „Trockenes“ festes Material wird zunächst mechanisch zerkleinert, anschließend in geeigneter Flüssigkeit gelöst oder suspendiert. Dazu dient zumeist physiologische Kochsalzlösung oder Pufferlösung, bei fettreichem Ausgangsprodukt auch Ethanol, Azeton oder Olivenöl. Bei geringer Löslichkeit des Ausgangsmaterials kann eine längerfristige Inkubation (z. B. über Nacht) mit der Lösungsflüssigkeit zweckmäßig sein. Durch Dialysieren oder Zentrifugieren können unterschiedliche Fraktionen flüssigen Testmaterials gewonnen werden. Bei Tests mit selbst präpariertem, nativem Material ist es wichtig, das Vorgehen bei Zubereitung und Anwendung genau zu dokumentieren. Dabei sind, soweit möglich, quantitative Angaben zu machen, beispielsweise über die Relation von Material zu Extraktionsflüssigkeit, Inkubationszeiten oder Verdünnungsreihen.

Beim „Prick-zu-Prick-Test“ wird mit der Lanzette in das allergenhaltige, nicht aufbereitete Material, meist ein Nahrungsmittel, gestochen und anschließend mit dieser Lanzette ein Prick-Test vorgenommen. Spuren des Materials, die an der Lanzettenspitze haften, können eine Testreaktion auslösen. Der Prick-zu-PrickTest wird vor allem in der Diagnostik der Nahrungsmittelallergie angewendet $[11,31]$.

\subsection{Kontrollen}

Zur Vermeidung grundsätzlicher Fehlinterpretationen sind stets dem gewählten Testverfahren entsprechende Kontrolltests durchzuführen: Ab dem Kleinkindesalter wird mit der $1 \%$ igen Histaminlösung ein Quaddeldurchmesser von $\geq 3 \mathrm{~mm}$ von fast allen Personen errreicht $[53,78]$. Als Positivkontrolle wird im
Pricktest daher 1,0\%iges, im Intrakutantest 0,01\%iges Histamindihydrochlorid mitgeführt [7]. Dabei ist zu beachten, dass mit der Histaminkontrolle nur eine Wirkung auf die Gefäße, nicht aber die Freisetzung von Mastzellmediatoren selbst überprüft wird, d. h. eine auf einer „Mastzellstabilisierung“ oder einer anders verursachten Areaktivität von Mastzellen beruhende verminderte Reagibilität wird nicht erfasst.

Als Negativkontrolle wird physiologische Kochsalzlösung verwendet. Enthält der kommerzielle Allergenextrakt Hilfsstoffe (z.B. Phenol zur Konservierung), so soll auch die Negativkontrolle diesen Hilfsstoff in gleicher Konzentration enthalten.

Beim Reibtest wird inertes Material, das dem Testmaterial hinsichtlich der Konsistenz weitgehend entspricht, z. B. ein mit physiologischer Kochsalzlösung getränkter Tupfer, mit vergleichbarer Intensität und Dauer auf der Haut gerieben.

\section{Ablesung und Dokumentation der Testreaktionen}

Die Ablesung der Sofortreaktion erfolgt nach 15-20 min [7,82]; auf der Haut verbliebenes Testmaterial wird vorher abgetupft. Die Ergebnisse bei sofortigem Entfernen der Extrakte (z.B. bei unruhigem Kind) sind vergleichbar mit solchen, bei denen die Testlösung nicht abgewischt wurde [62].

Erfasst werden bei der Ablesung Erythem und Quaddel; letztere kann durch leichtes Spannen der Haut besser sichtbar gemacht werden.

Es gibt verschiedene Methoden, die Testreaktion zu dokumentieren. Als positive Testreaktion gilt beim Pricktest ein mittlerer Quaddeldurchmesser von $\geq 3 \mathrm{~mm}$ oder eine Quaddelfläche von $\geq 9 \mathrm{~mm}^{2}$ [22], beim Intrakutantest ein Quaddeldurchmesser von $\geq 5 \mathrm{~mm}[22,58]$. Dieser wird ermittelt durch die Summe aus dem größten Durchmesser und dem größten hierzu senkrechten Durchmesser (in mm) geteilt durch zwei. Für wissenschaftliche Zwecke sind planimetrische Verfahren angezeigt. Für Arzneistoffe wurden für die Ablesung der Intrakutantestreaktion andere Kriterien zugrundegelegt [10]: Erst wenn der Durchmesser der bei Tests gesetzten Quaddel um $3 \mathrm{~mm}$ zunimmt und eine Rötung sichtbar ist, gilt die Testreaktion als positiv.

Am exaktesten ist die metrische Dokumentation des Quaddeldurchmessers, die als Standard zur Dokumentation der Testreaktionen empfohlen wird. Wenn die Hauttestreaktionen mittels eines Bewertungsschemas abgelesen werden, muss dessen Definition aus der Befunddokumentation hervorgehen. In der Praxis hat sich das in $\bullet$ Tab. 8 gezeigte Bewertungsschema für Prickund Intrakutantestreaktionen bewährt [modifiziert nach 72]. Nachteil einer semiquantitativen Ablesung ist eine schlechte Übereinstimmung der Ergebnisse unterschiedlicher Untersucher [51].

Tab. 8 Semiquantitatives Bewertungsschema von Prick- und Intradermaltest anhand des mittleren Quaddeldurchmessers (modifiziert nach [72]).

$\begin{array}{lll}\text { Beurteilung } & \text { Prick }(\mathbf{m m} \varnothing) & \text { Intradermal }(\mathbf{m m} \varnothing) \\ \varnothing & 0 & 0 \\ (+) & <3 & <5 \\ + & \geq 3-<4 & \geq 5-<8 \\ ++ & \geq 4-<5 & \geq 8-<11 \\ +++ & \geq 5-<6 & \geq 11-<15 \\ ++++ & \geq 6 & \geq 15\end{array}$

$\varnothing$, negativ; (+), fraglich positive; +, einfach positiv; ++, zweifach positiv; +++, dreifach positiv; ++++ , vierfach positiv. 
Da die individuelle Reagibilität auf Histamin sehr unterschiedlich ist, kann eine Bewertung der Testreaktionen in Relation zur Histaminquaddel nicht empfohlen werden. So fallen bei Kleinkindern die Quaddelgrößen auf Histamin kleiner aus, auf Haut dunkel pigmentierter Personen dagegen größer [7]. Bei manchen Patienten, vor allem solchen mit schwereren atopischen Erkrankungen, ist das Erythem einer Testreaktion nur schwach ausgeprägt oder fehlt vollständig (Korrelat zum „weißen Dermographismus“), insbesondere ist die Dosis-Wirkungskurve individuellen Schwankungen unterworfen.

Sämtliche Testergebnisse werden in einem Befundbogen notiert. Name des Patienten, Name und Praxis- bzw. Klinikadresse des Untersuchers, Datum der Untersuchung und Ablesezeitpunkt sind zu dokumentieren. Aus dem Befundbogen sollen weiter die verwendeten Testextrakte (Hersteller), die Methode und das Beurteilungsschema der Ablesung hervorgehen. Auch alle negativen Resultate sind eindeutig als solche zu verzeichnen.

Üblicherweise erfolgt nur eine Ablesung nach 15-20 min (Sofortablesung). Zur Erfassung von verzögerten Reaktionen oder Spätreaktionen werden bei entsprechender Fragestellung weitere Ablesungen nach etwa $6-8,24$ und 48 h, gegebenenfalls auch noch später, vorgenommen. Im Übrigen sollte der Patient angewiesen werden, verzögerte Reaktionen bei Wiedervorstellung zu berichten. Falls erforderlich, wird der Test dann mit geeigneten Ablesezeitpunkten wiederholt. Die Dokumentation von verzögerten Reaktionen erfolgt durch Angabe des größten sowie des größten hierzu senkrechten Durchmessers von Erythem und Induration (in Millimetern) sowie der morphologischen Beschreibung, z. B. Papeln, Bläschen oder Schuppung.

Eine Therapie der Testreaktionen ist meist nicht nötig, da die Symptome Pruritus und Erythem meist rasch wieder abklingen. Nicht hilfreich ist eine topische Anwendung von Glukokortikoiden [37], bei Bedarf können kühlende Gels versucht werden.

\section{Beurteilung}

$\nabla$

Diagnostisch verwertbar sind Testergebnisse nur, wenn auf die Negativkontrolle keine (maximaler Quaddeldurchmesser beim Pricktest $<2 \mathrm{~mm}$ ), auf die Histaminkontrolle eine eindeutige mindestens 1+Reaktion (Quaddeldurchmesser beim Pricktest mit mindestens $3 \mathrm{~mm}$, beim Intrakutantest mindestens $5 \mathrm{~mm}$ Durchmesser) auftritt.

Wenn gegen ein häufig zu IgE-vermittelten Reaktionen führendes kommerzielles Allergen eine mindestens 1+Reaktion auftritt, ist damit die IgE-bedingte Auslösung wahrscheinlich. Wenn mit selbst präparierten Zubereitungen getestet wird, ist dies erheblich kritischer zu sehen. Kommt es hier zu einer Reaktion, so sind Kontrolltests an einer ausreichenden Anzahl von Personen erforderlich, um unspezifische (toxische) Reaktionen auszuschließen.

Die klinische Bedeutung von Hauttestreaktionen ist sorgfältig zu überprüfen, anhand der gegebenenfalls zu ergänzenden Anamnese, des Ergebnisses der Bestimmung spezifischer IgE-Antikörper und anhand von Provokationstests. Zwar kommt starken Hauttestreaktionen vergleichsweise häufiger eine klinische Relevanz zu als schwächeren Reaktionen [85]. Aufgrund eines großen Quaddeldurchmessers auf die klinische Relevanz zu schließen, ist aber ebenso wenig zulässig, wie eine schwache Testreaktion als irrelevant einzuschätzen.

Wenn spezifische Testreaktionen reproduzierbar, jedoch ohne klinische Relevanz sind, ist hier nicht von „falsch positiven“ Reak- tionen, sondern von einer klinisch nicht relevanten Sensibilisierung zu sprechen. Auch verzögerte Reaktionen bei Hauttests sind in ihrer diagnostischen Wertigkeit nicht anders zu beurteilen als Soforttyp-Reaktionen, das heißt, sie können nicht ohne weiteres als klinisch relevant angesehen werden. Im Allergiepass werden Sensibilisierungen ohne klinische Bedeutung nicht verzeichnet.

Wirklich „falsch positive“ Reaktionen treten bei urtikariellem Dermographismus auf. Urticae an der Teststelle können sich dabei auch erst einige Stunden später einstellen (Urticaria factitia tarda). Typisch sind Reaktionen in (fast) allen Testfeldern, die dann oft auch weitgehend monomorph imponieren. Eine Auswertung eines solchen Tests ist nicht möglich. Eine Wiederholung des Tests nach einigen Wochen erbringt aber nicht selten verwertbare Ergebnisse.

„Falsch positive“ Reaktionen können auch durch technische Fehler, z.B. durch zu geringe Abstände zwischen den Testfeldern oder ungenügendes Abwischen der Lanzette, bedingt sein. Treten Reaktionen auf Allergene trotz negativer Histaminkontrolle auf, so ist die korrekte Durchführung des Tests zweifelhaft. Bei allen widersprüchlichen oder „ungewöhnlichen“ Ergebnissen ist der Test teilweise oder ganz zu wiederholen.

Trotz eindeutiger Anamnese einer Allergie vom Soforttyp kann eine Reaktion im Hauttest ausbleiben. Wenn auch In-vitro-Tests nicht weiterführend waren, ist zunächst zu überprüfen, ob die Tests technisch korrekt mit allen infrage kommenden Allergenen und unter Ausnutzung der verfügbaren Testmethoden ausgeführt wurden, z.B. Tests mit nativem Material oder gegebenenfalls Intrakutantest. Jedoch finden sich auch bei bestehender Allergie keineswegs immer entsprechende Hauttestreaktionen, vor allem bei längerem zeitlichen Abstand zur letzten Allergenexposition oder bei Patienten ohne (eindeutige) atopische Veranlagung. Auch ist bei negativem Hauttest die Möglichkeit einer nur auf das erkrankte Organ beschränkten Präsenz spezifischer IgEAntikörper zu bedenken; dies kann gebenenfalls durch organspezifische Untersuchungen nachgewiesen werden. Tests, die zu nicht schlüssigen Ergebnissen geführt haben, sollten nach sorgfältiger Elimination von möglicherweise verfälschenden Einflüssen wiederholt werden. Beispielsweise sind gelegentlich in der Frühphase einer respiratorischen allergischen Erkrankung Reaktionen im Hauttest auf das auslösende Allergen noch nicht zu finden und treten erst später auf.

Aus einer klinisch nicht relevanten Sensibilisierung ergeben sich im allgemeinen keine allergenspezifischen praktischen Konsequenzen wie gezielte Karenz, prophylaktische Pharmakotherapie oder gar Hyposensibilisierung. Der Patient ist aber auf das Bestehen einer atopischen Diathese und die sich daraus ergebenden Konsequenzen hinzuweisen.

Datum der letzten Überarbeitung: 19. 10. 2009

Nächste Überprüfung geplant: 2012

Verfahren zur Leitlinien-Entwicklung:

Literaturrecherche, Konsensuskonferenzen mit Moderation durch Priv.-Doz. Dr. H. Sitter

\section{Interessenkonflikt}

\section{$\nabla$}

F. Ruëff hat Reisekosten und Vortragshonorare von ALK-Abelló, Bencard und HAL erhalten und erhält eine finanzielle Unterstützung für ein Forschungsvorhaben von ALK-Abelló und Phadia. 
K. Brockow hat für Beratertätigkeit, wissenschaftliche Vorträge von folgenden Arzneimittelherstellern Zuwendungen erhalten: CT Arzneimittel, Bayer, ALK-Abelló, Allergopharma, Merckle Recordati.

K.-C. Bergmann hat Vortragshonorare erhalten von und sich an klinischen Prüfungen beteiligt für folgende Firmen: ALK-Abelló, Bencard Allergie, Boehringer Ingelheim, Chiesi, Essex Pharma, HAL Allergie, Novartis Pharma Arzneimittel, Stallergenes.

T. Fuchs hat Vortragshonorare von ALK-Abelló, Bencard Allergie $\mathrm{GmbH}$, HAL Allergie, Stallergenes und Honorare für Beratertätigkeit von Stallergenes, Bencard erhalten.

K. Jung hat Drittmittel für Forschungsvorhaben von ALK-Abelló und Honorare für Vortragstätigkeiten von den Unternehmen ALK-Abelló und Bencard Allergie erhalten.

L. Klimek hat für Beratertätigkeit, wissenschaftliche Vorträge und/oder die Teilnahme an klinischen Prüfungen von folgenden Arzneimittelherstellern Zuwendungen erhalten: ALK-Abelló, Allergopharma, Artu Biologicals, Bencard Allergie, Boehringer Ingelheim, CYTOS-Biotechnology, Curalogic, MSD/Essex Pharma, GSK, HAL Allergie, Hartington, LETI, Lofarma, Merckle Recordati, Novartis, Phadia, Roxall, Stallergenes.

O. Pfaar hat für Beratertätigkeit und/oder wissenschaftliche Vorträge und/oder die Teilnahme an klinischen Prüfungen von folgenden Arzneimittelherstellern Zuwendungen erhalten: Allergopharma, ALK-Abelló, HAL-Allergy, Bencard, GlaxoSmithKline (GSK), Eumecom, MSD/Essex-Pharma, Hartington, Stallergenes, Roxall, Lofarma, Artu Biologicals, Febena PHARMA, Optima Pharmaceutische GmbH, CYTOS-Biotechnology, Curalogic, Novartis und LETI.

B. Przybilla hat für wissenschaftliche Vorträge und/oder die Beteiligung an klinischen Prüfungen finanzielle Zuwendungen erhalten von ALK-Abelló, Phadia, Stallergenes und Novartis.

$\mathrm{H}$. Müsken hat für eine Beratertätigkeit und Vorträge Zuwendungen erhalten von Bencard Allergie sowie Vortragshonorare von Astellas, HAL Allergie, LETI, Essex Pharma, AstraZeneca und Chiesi.

W. Wehrmann hat Vortragshonorare von ALK-Abelló, Allergopharma und Stallergenes sowie Honorare für Beratertätigkeit von ALK-Abelló und Novartis erhalten.

$\mathrm{H}$. Sitter gibt an, dass keine Interessenkonflikte bestehen.

\section{Literatur}

1 Abramowitz PW, Perez MM, Johnson CE et al. Effect of theophylline, terbutaline, and their combination on the immediate hypersensitivity skin-test reaction. J Allergy Clin Immunol 1980; 66: 123- 128 (Evidenzlevel 1b)

2 Adinoff AD, Rosloniec DM, McCall LL, Nelson HS. A comparison of six epicutaneous devices in the performance of immediate hypersensitivity skin testing. J Allergy Clin Immunol 1989; 84: 168-174 (Evidenzlevel 2b)

3 Allert M-H, Enzmann H. Schmerzloser Pricktest durch die Verwendung von EMLA? Allergologie 1995; 18: 429-432 (Evidenzlevel 3)

4 Bergmann K-C, Müsken H. Kutane Tests. In: Przybilla B, Bergmann K-C, Ring J, Hrsg. Praktische allergologische Diagnostik. Darmstadt: Steinkopff-Verlag; 2000: 9-22 (Evidenzlevel 4)

5 Berkowitz RB, Tinkelman DG, Lutz C et al. Evaluation of the Multi-Test device for immediate hypersensitivity skin testing. J Allergy Clin Immunol 1992; 90: 979-985 (Evidenzlevel 2b)

6 Bernstein DI, Wanner M, Borish L, Liss GM. Immunotherapy Committee, American Academy of Allergy, Asthma and Immunology. Twelve-year survey of fatal reactions to allergen injections and skin testing: 1990 - 2001. J Allergy Clin Immunol 2004; 113: 129-1136 (Evidenzlevel 4)

7 Bernstein IL, Li JT, Bernstein DI et al. American Academy of Allergy, Asthma and Immunology; American College of Allergy, Asthma and Immuno- logy. Allergy diagnostic testing: an updated practice parameter. Ann Allergy Asthma Immunol 2008; 100 (Suppl. 3): 1 -148 (Evidenzlevel 4)

8 Bonnekoh B, Merk HF. Safety of latex prick skin testing in allergic patients. JAMA 1992; 267: 2603-2604 (Evidenzlevel 4)

9 Bousquet J, Michel F-B. Precision of prick and puncture tests. J Allergy Clin Immunol 1992; 90: 870-872 (Evidenzlevel 4)

10 Brockow $K$, Romano A, Blanca $M$ et al. General considerations for skin test procedures in the diagnosis of drug hypersensitivity. Allergy 2002; 57: 45 - 51 (Evidenzlevel 4)

11 Cantani A, Micera M. The prick by prick test is safe and reliable in 58 children with atopic dermatitis and food allergy. Eur Rev Med Pharmacol Sci 2006; 10: 115 - 120 (Evidenzlevel 3)

12 Carr WW, Martin B, Howard RS et al. Immunotherapy Committee of the American Academy of Allergy, Asthma and Immunology. Comparison of test devices for skin prick testing. J Allergy Clin Immunol 2005; 116: 341 - 346 (Evidenzlevel 2b)

13 Chanal I, Horst M, Segalen C et al. Comparison between modified skin prick test with standardized allergen extracts and Phazet. J Allergy Clin Immunol 1988; 82: 878-881 (Evidenzlevel 3)

14 Christensen M, Moelby L, Svendsen F. Reliability of skin prick tests during terfenadine treatment in adults with pollen rhinitis. Allergy 1994; 49: 702 - 706 (Evidenzlevel 1b)

15 Corder WT, Wilson NW. Comparison of three methods of using the DermaPIK with the standard prick method for epicutaneous skin testing. Ann Allergy Asthma Immunol 1995; 75: 434 - 438 (Evidenzlevel 3)

16 Cuhadaroglu C, Erelel M, Kiyan E et al. Role of Zafirlukast on skin prick test. Allergol Immunopathol (Madr) 2001; 29: 66 -68 (Evidenzlevel 2b)

17 Demoly P, Bousquet J, Manderscheid J-C et al. Precision of skin prick and puncture tests with nine methods. J Allergy Clin Immunol 1991; 88: $758-762$ (Evidenzlevel 2a)

18 Demoly P, Piette V, Bousquet J. In vivo methods for study of allergy. In: Adkinson Jr. NF, Yunginger JW, Busse WW et al. eds. Middleton's Allergy - Principles and practice. 6th edition. Philadelphia: Mosby; 2003: 430 - 439 (Evidenzlevel 4)

19 Des Roches A, Paradis L, Bougeard YH et al. Long-term oral corticosteroid therapy does not alter the results of immediate-type allergy skin prick tests. J Allergy Clin Immunol 1996; 98: 522 - 527 (Evidenzlevel 2b)

20 Devenney I, Fälth-Magnusson K. Skin prick tests may give generalized allergic reactions in infants. Ann Allergy Asthma Immunol 2000; 85: 457-460 (Evidenzlevel 3)

21 Dreborg S, Holgersson M, Nilsson G et al. Dose response relationship of allergen, histamine, and histamine releasers in skin prick test and precision of the skin prick test method. Allergy 1987; 42: 117-125 (Evidenzlevel 3)

22 Dreborg S, Frew A. EAACI Subcommittee on Skin tests. Position paper: Allergen standardization and skin tests. Allergy 1993; 48 (Suppl.): 48 82 (Evidenzlevel 4)

23 Dreborg S. The risk of general reactions to skin prick testing (SPT). Allergy 1996; 51: 60 - 61 (Evidenzlevel 4)

24 Dreborg S. Histamine reactivity of the skin. Allergy 2001; 56: 359-364 (Evidenzlevel 4)

25 Dundas I, Chan E, McKenzie SA. Faster, simpler skin-prick testing. J Paediatr Child Health 2005; 41: 695-696 (Evidenzlevel 2b)

26 Fisher MM, Bowey CJ. Intradermal compared with prick testing in the diagnosis of anaesthetic allergy. Br J Anaesth 1997; 79: 59- 63 (Evidenzlevel 3)

27 Fritsché R. Animal models in food allergy: assessment of allergenicity and preventive activity of infant formulas. Toxicol Lett 2003; 140 141: 303 - 309 (Evidenzlevel 4)

28 Goldberg A, Confino-Cohen R. Timing of venom skin tests and IgE determinations after insect sting anaphylaxis. J Allergy Clin Immunol 1997; 100: 182 - 184 (Evidenzlevel 3)

29 Gronemeyer W, Debelic M. Der sogenannte „Reibtest“, seine Anwendung und klinische Bedeutung. Dermatologica 1967; 134: 208- 218 (Evidenzlevel 4)

30 Heinzerling L, Frew AJ, Bindslev-Jensen C et al. Standard skin prick testing and sensitization to inhalant allergens across Europe - a survey from the GALEN network. Allergy 2005; 60: 1287-1300 (Evidenzlevel 2a)

31 Henzgen M, Ballmer-Weber B, Erdmann S et al. Hauttestungen mit Nahrungsmittelallergenen. Allergo J 2008; 17: 401 - 406 (Evidenzlevel 4)

32 Hill 3rd SL, Krouse JH. The effects of montelukast on intradermal wheal and flare. Otolaryngol Head Neck Surg 2003; 129: 199- 203 (Evidenzlevel 1b) 
33 Hutin Y, Hauri A, Chiarello L et al. Injection Safety Best Practices Development Group. Best infection control practices for intradermal, subcutaneous, and intramuscular needle injections. Bull World Health Organ 2003; 81: 491 - 500 (Evidenzlevel 4)

34 Indrajana T, Spieksma FT, Voorhorst R. Comparative study of the intracutaneous, scratch and prick tests in allergy. Ann Allergy 1971; 29: 639 - 650 (Evidenzlevel 3)

35 Jeep S, Reiprich G, Kunkel G. Yellow jacket allergy. Comparison of skin prick tests and intradermal tests with three different yellow jacket venom extracts. Allergy 1992; 47: 35 - 40 (Evidenzlevel 3)

36 Kaplan AP, Anderson JA, Valentine MD et al. Beta-adrenergic blockers, immunotherapy, and skin testing. American Academy of Allergy and Immunology. J Allergy Clin Immunol 1989; 84: 129-130 (Evidenzlevel 4)

37 Kelso JM. Application of topical corticosteroids to sites of positive immediate-type allergy skin tests to relieve itching: results of a doubleblind, placebo-controlled trial. Ann Allergy Asthma Immunol 2007; 98: 182 - 184 (Evidenzlevel 1b)

38 Kemeny DM, Lambourn EM, Patel S et al. Comparison of a new type of skin test (Phazet) with existing skin test methods and the radioallergosorbent test (RAST). Clin Exp Allergy 1989; 19: 613 - 617 (Evidenzleve 2b)

39 King MJ, Lockey RF. Allergen prick-puncture skin testing in the elderly. Immunopharmacol Immunotoxicol 2000; 22: 253- 266 (Evidenzlevel 4)

40 Klimek L, Bachert C, Schlenter W. Die nasale Provokationstestung. Allergo J 2001; 10: 396 - 405 (Evidenzlevel 4)

41 Koepke JW, Reller LB, Masters HA et al. Viral contamination of intradermal skin test syringes. Ann Allergy 1985; 55: 776 - 778 (Evidenzlevel 4)

42 Koller DY, Pirker C, Jarisch $R$ et al. Influence of the histamine control on skin reactivity in skin testing. Allergy 1992; 47: 58 - 59 (Evidenzlevel 3)

43 Kunz R, Ollenschlager G, Raspe $\mathrm{H}$ et al. Evidenzbasierte Medizin in Klinik und Praxis. Köln: Deutscher Ärzte-Verlag; 2007 (Evidenzlevel 4)

44 Lockey RF, Benedict LM, Turkeltaub PC et al. Fatalities from immunotherapy) and skin testing. J Allergy Clin Immunol 1987; 79: 660-677 (Evidenzlevel 4)

45 Lüderitz-Püchel U, Keller-Stanislawski B, Haustein D. Neubewertung des Risikos von Test- und Therapieallergenen. Eine Analyse der UAW-Meldungen von 1991 bis 2000. Bundesgesundheitsbl Gesundheitsforsch Gesundheitsschutz 2001; 44: 709 - 718 (Evidenzlevel 3)

46 Lutz CT, Bell CE, Wedner HJ et al. Allergy testing of multiple patients should no longer be performed with a common syringe. N Engl J Med 1984; 310: 1335 - 1337 (Evidenzlevel 4)

47 Malling H-J. Methods of skin testing. Allergy 1993; 48 (Suppl. 14): 55 56 (Evidenzlevel 4)

48 Malling $H-J$, Weeke B. Position paper: Immunotherapy. Allergy 1993 ; 48 (Suppl. 14): 9 - 35 (Evidenzlevel 4)

49 Maurer T. Guinea pigs in hypersensitivity testing. Methods 2007; 41: 48-53 (Evidenzlevel 4)

50 Mazer BD, Al-Tamemi S, Yu JW et al. Immune supplementation and immune modulation with intravenous immunoglobulin. J Allergy Clin Immunol 2005; 116: 941 - 944 (Evidenzlevel 4)

51 McCann WA, Ownby DR. The reproducibility of the allergy skin test scoring and interpretation by board-certified/board-eligible allergists. Ann Allergy Asthma Immunol. 2002; 89: 368 - 371 (Evidenzlevel 3)

52 McKay SP, Meslemani D, Stachler RJ et al. Intradermal positivity after negative prick testing for inhalants. Otolaryngol Head Neck Surg 2006; 135: 232 - 235 (Evidenzlevel 3)

53 Ménardo JL, Bousquet J, Rodière $M$ et al. Skin test reactivity in infancy. J Allergy Clin Immunol 1985; 75: 646-651 (Evidenzlevel 3)

54 Munro CS, Higgins EM, Marks JM et al. Cyclosporin A in atopic dermatitis: therapeutic response is dissociated from effects on allergic reactions. Br J Dermatol 1991; 124: 43 - 48 (Evidenzlevel 3)

55 Nelson HS, Rosloniec DM, McCall LI et al. Comparative performance of five commercial prick skin test devices. J Allergy Clin Immunol 1993; 92: 750 - 756 (Evidenzlevel 2b)

56 Nelson HS, Knoetzer J, Bucher B. Effect of distance between sites and region of the body on results of skin prick tests. J Allergy Clin Immunol 1996; 97: 596 - 601 (Evidenzlevel 2b)

57 Niemeijer NR, Kauffman HF, van Hove W et al. Effect of dilution, temperature, and preservatives on the long-term stability of standardized inhalant allergen extracts. Ann Allergy Asthma Immunol 1996; 76: 535 - 540 (Evidenzlevel 2b)

58 Norman PS. In vivo methods of study of allergy: skin and mucosal technique and interpretation. In: Middleton E, Ellis EF, Reed CE, eds. Aller- gy: Principles and Practice. 2nd edition. St. Louis: Mosby; 1982: 295 302 (Evidenzlevel 4)

59 Novembre E, Bernardini R, Bertini $G$ et al. Skin-prick-test-induced anaphylaxis. Allergy 1995; 50: 511 - 513 (Evidenzlevel 4)

60 Osterballe M, Scheller $R$, Stahl Skov P et al. Diagnostic value of scratchchamber test, skin prick test, histamine release and specific IgE in birch-allergic patients with oral allergy syndrome to apple. Allergy 2003; 58: 950 - 953 (Evidenzlevel 2b)

61 Østerballe O, Weeke B. A new lancet for skin prick testing. Allergy 1979; 34: 209 - 212 (Evidenzlevel 2b)

62 Ownby DR, Anderson JA. An improved skin-test procedure for young children. J Allergy Clin Immunol 1982; 69: 533 - 535 (Evidenzlevel 2b)

63 Petersen LJ, Skov PS. The effect of salmeterol and salbutamol on mediator release and skin responses in immediate and late phase allergic cutaneous reactions. Inflamm Res 1999; 48: 527-532 (Evidenzlevel 2b)

64 Petersen LJ, Skov PS. Effect of terbutaline and bambuterol on immediate-type allergic skin responses and mediator release in human skin. Inflamm Res 2003; 52: 372 - 377 (Evidenzlevel 1b)

65 Piette V, Bourret E, Bousquet J et al. Prick tests to aeroallergens: is it possible simply to wipe the device between tests? Allergy 2002; 57 : 940 - 942 (Evidenzlevel 2a)

66 Pipkorn U, Andersson M. Topical dermal anaesthesia inhibits the flare but not the wheal response to allergen and histamine in the skin prick test. Clin Allergy 1987; 17: 307 - 311 (Evidenzlevel 2b)

67 Pipkorn U, Hammarlund A, Enerbäck L. Prolonged treatment with topical glucocorticoids results in an inhibition of the allergen-induced weal-and-flare response and a reduction in skin mast cell numbers and histamine content. Clin Exp Allergy 1989; 19: 19-25 (Evidenzlevel 1b)

68 Rancé F, Juchet A, Brémont F et al. Correlations between skin prick tests using commercial extracts and fresh foods, specific IgE, and food challenges. Allergy 1997; 52: 1031 - 1035 (Evidenzlevel 3)

69 Rasp G. Pricktest: Nadel oder Lanzette? Allergologie 1991; 12: 480 482 (Evidenzlevel 3)

70 Reid MJ, Lockey RF, Turkeltaub PC et al. Survey of fatalities from skin testing and immunotherapy 1985 - 1989. J Allergy Clin Immunol 1993 92: 6-15 (Evidenzlevel 3)

71 Renz H, Becker W-M, Bufe A et al. In-vitro-Allergiediagnostik. Gemeinsames Positionspapier der DGAKI und der DDG. Allergo Journal 2002; 11: 492 - 506 (Evidenzlevel 4)

72 Ring J. Angewandte Allergologie. München: MMV Verlag; 1992 (Evidenzlevel 4)

73 Ring J, Brockow K, Duda D et al. Akuttherapie anaphylaktischer Reaktionen. Leitlinie. Allergo J 2007; 16: 420 - 434 (Evidenzlevel 4)

74 Rosen JP, Selcow JE, Mendelson LM et al. Skin testing with natural foods in patients suspected of having food allergies: is it a necessity? J Allergy Clin Immunol 1994; 93: 1068 - 1070 (Evidenzlevel 3)

75 Scherer K, Grize L, Schindler C et al. Reaction pattern to histamine and codeine in a human intradermal skin test model. Clin Exp Allergy 2007; 37: 39-46 (Evidenzlevel 2b)

76 Schnuch A, Aberer W, Agathos $M$ et al. Leitlinien der Deutschen Kontaktallergie-Gruppe (DKG) zur Durchführung des Epikutantests mit Kontaktallergenen. Hautarzt 2001; 52: 864 - 866 (Evidenzlevel 4)

77 Simons FER, Gillespie GA, Simons KJ. Local anaesthetic creams and intradermal skin tests. Lancet 1992; 339: 1351 - 1352 (Evidenzlevel 1b)

78 Skassa-Brociek W, Manderscheid J-C, Michel F-B et al. Skin test reactivity to histamine from infancy to old age. J Allergy Clin Immunol 1987; 80: 711 - 716 (Evidenzlevel 3)

79 Spector SL. Effect of a selective beta 2 adrenergic agonist and theophylline on skin test reactivity and cardiovascular parameters. J Allergy Clin Immunol 1979; 64: 23 - 28 (Evidenzlevel 2b)

80 Stefanoff $V$. Non-fatal anaphylaxis caused by ampicillin scratch-test Report of case. Int J Oral Maxillofac Surg 1989; 18: 17 (Evidenzlevel 4)

81 Turkeltaub $P C$, Gergen $P$. The risk of adverse reactions from percutaneous prick-puncture allergen skin testing, venipuncture, and body measurements: data from the second National Health and Nutrition Examination Survey 1976 - 80 (NHANES II). J Allergy Clin Immunol 1989; 84: 886 - 890 (Evidenzlevel 3)

82 Van Metre TE, Adkinson NF, Kagey-Sobotka A et al. How should we use skin testing to quantify IgE sensitivity. J Allergy Clin Immunol 1990; 86: 583 - 586 (Evidenzlevel 4)

83 Wood RA, Phipatanakul W, Hamilton RG et al. A comparison of skin prick tests, intradermal skin tests, and RASTs in the diagnosis of cat allergy. J Allergy Clin Immunol 1999; 103: 773 - 779 (Evidenzlevel 3) 